\title{
Manifold Studies of Nonlinear Antenna Array Geometries
}

\author{
Athanassios Manikas, Member, IEEE, Adham Sleiman, Student Member, IEEE, and Ioannis Dacos
}

\begin{abstract}
In this paper, the manifold of a general two- or threedimensional (2-D or 3-D) array of sensors is studied using differential geometry. By considering the azimuth and elevation angles as the parameters of interest, a manifold surface is formed embedded in a multidimensional complex space. Initially, this surface is investigated by establishing a number of differential geometry parameters associated with the array manifold. Then, the concept of $d e$ velopment is proposed for mapping manifold surfaces (embedded in a multidimensional complex space) on the real 2-D parameter plane. The proposed mapping preserves certain differential geometry characteristics of the manifold surface and provides a simplified representation for the analysis. The potential benefits of this mapping, as well as of the proposed parameters, are demonstrated in the analysis of 3-D and planar arrays of omnidirectional sensors and in a number of potential applications, varying from array design to handling the array ambiguity problem.
\end{abstract}

Index Terms-Array manifold, array processing, differential geometry, direction finding, nonlinear arrays.

\section{INTRODUCTION}

A RRAY systems for signal detection, direction finding, superresolution beamforming, etc., are strongly dependent on the properties and parameters of the array manifold [1]-[7]. Therefore, a rigorous analysis of the array manifold is essential and can be accomplished effectively through the study of the manifold's differential geometry. Thus far, this analysis has been carried out for one-dimensional (1-D) manifolds, associated with linear arrays, which are curves embedded in a multidimensional complex space. These 1-D manifolds (curves) have been found to have a hyperhelical shape, the properties of which have been extensively studied [1], [4], [8]. For array systems that involve both azimuth and elevation angles as parameters of interest, the array manifold is a surface rather than a curve and is associated with nonlinear array structures. Thus far, the investigation of manifold surfaces has consistently been done by breaking them down into lines and treating those lines as manifold curves of equivalent linear arrays (ELAs). However, advanced insight into arrays may be provided by considering the

Manuscript received October 29, 1999; revised November 7, 2000. The associate editor coordinating the review of this paper and approving it for publication was Dr. Kristine Bell.

A. Manikas and A. Sleiman are with the Communications and Signal Processing Research Group, Department of Electrical and Electronic Engineering, Imperial College of Science Technology and Medicine, University of London, London, U.K. (e-mail: a.manikas@ic.ac.uk).

I. Dacos was with Communications and Signal Processing Research Group, Department of Electrical and Electronic Engineering, Imperial College of Science Technology and Medicine, University of London, London, U.K. He is now with Greek Telecom (OTE), Athens, Greece, and the Communication Engineering Department, Hellenic Air Force Academy, Athens, Greece.

Publisher Item Identifier S 1053-587X(01)01418-0. manifold surface as a ngle entity, and for this reason, differential geometry for surfaces rather than curves should be employed. In this paper, the manifold surface of a nonlinear array of sensors has been analyzed using differential geometry [9]-[11], and its main parameters have been identified. The essential parameters have been grouped into those related to points on the surface and those related to curves on the surface. These characteristics should be maintained in any simplified representation of the manifold, and therefore, in this paper, we propose a mapping of the manifold surface (embedded in a multidimensional complex space) onto the real plane (2-D space), which preserves these characteristics under certain conditions. The potential benefits of this mapping, as well as of the proposed parameters, are demonstrated in the analysis of 3-D and planar arrays of omnidirectional sensors and in a number of potential applications, varying from array design to handling the array ambiguity problem.

Based on the above discussion, the paper is organized as follows. In Section II, the most important differential geometry related parameters of the array manifold are identified, and their analytical expressions are presented. In Section III, the concept of the development is proposed, which is a transformation, mapping the complicated manifold surface onto a real plane. The proposed theoretical framework is applied in Section IV to array structures of omnidirectional sensors and is used in Section V to demonstrate its utilization in a number of potential applications. Finally, in Section VI, the paper is concluded.

\section{DifFerential GeOMetry Parameters of the Surface}

In an azimuth $\theta$ and elevation $\phi$ direction finding array system of $N$ sensors, the manifold vector $\underline{a}(\theta, \phi)$ is defined as

$$
\underline{a}(\theta, \phi)=\underline{\gamma}(\theta, \phi) \odot \exp \left(-j \underline{\underline{r}}^{T} \underline{k}\right)
$$

where $\underline{\underline{r}}=\left[\underline{r}_{x}, \underline{r}_{y}, \underline{r}_{z}\right]^{T} \in R^{3 \times N}$ is the sensor position matrix in half wavelengths with the array centroid taken as the array reference point $(0,0,0)$ (i.e., $\operatorname{sum}\left(\underline{r}_{x}\right)=\operatorname{sum}\left(\underline{r}_{y}\right)=$ $\left.\operatorname{sum}\left(\underline{r}_{z}\right)=0\right), \underline{k}=\pi[\cos \phi \cos \theta, \cos \phi \sin \theta, \quad \sin$ $\phi]^{T} \in R^{3 \times 1}$ denotes the wavenumber vector, $\odot$ represents the Hadamard product, and $\gamma(\theta, \phi) \in C^{N \times 1}$ is the vector with elements of the directional gains of the sensors.

The locus of all manifold vectors $a(\theta, \phi) \forall(\theta, \phi)$ forms a surface known as the array manifold. This is a 2-D manifold i.e., a 2-D nonlinear complex subspace (function of two variables), embedded in an $N$-dimensional complex space. For a point $(\theta, \phi)$ on the manifold surface, four differential geometry parameters of interest intrinsic to the manifold surface have been identified in the literature and extended from 3-D real space to 
$N$-dimensional complex space. In order to achieve this, we have taken into consideration all the ramifications and subtleties involved in this process. These parameters are the manifold metric $\mathbb{G}$, which is a function of the derivatives of the manifold vector of (1), and the Gaussian curvature $K$, which is a function of the manifold metric $\mathbb{G}$.

If the point $(\theta, \phi)$ on the manifold surface is also taken as a point on a curve on the manifold surface, then the following two parameters, which are also functions of the metric $\mathbb{G}$, are of interest:

- the arc length $s$ of the curve up to that point;

- the geodesic curvature $\kappa_{g}$ at that point.

\section{A. Manifold Metric}

The manifold metric is a $2 \times 2$ real matrix with elements known in differential geometry as the first fundamental coefficients (or metric coefficients) and is defined as follows:

$$
\begin{aligned}
\mathbb{G}=\operatorname{Re}\left\{\mathbb{T}^{H} \mathbb{T}\right\} & =\left[\begin{array}{cc}
\left\|\underline{a}_{\theta}\right\|^{2}, & \operatorname{Re}\left\{\underline{a}_{\theta}^{H} \underline{a}_{\phi}\right\} \\
\operatorname{Re}\left\{\underline{a}_{\phi}{ }^{H} \underline{a}_{\theta}\right\}, & \left\|\underline{a}_{\phi}\right\|^{2}
\end{array}\right] \\
& =\left[\begin{array}{ll}
g_{\theta \theta}, & g_{\theta \phi} \\
g_{\phi \theta}, & g_{\phi \phi}
\end{array}\right]
\end{aligned}
$$

where the matrix $\mathbb{T}=\left[\underline{a}_{\theta}, \underline{a}_{\phi}\right] \in C^{N \times 2}$ represents the tangent plane $^{1}$ with

$$
\underline{a}_{\theta}=\frac{d \underline{a}(\theta, \phi)}{d \theta} \in C^{N \times 1} \text { and } \underline{a}_{\phi}=\frac{d \underline{a}(\theta, \phi)}{d \phi} \in C^{N \times 1} .
$$

The metric $\mathbb{G}$ is a semipositive definite symmetric matrix. Its elements are called the first fundamental coefficients or metric coefficients and entirely describe the manifold properties of the surface. For instance, the angle between the two parameter curves of the surface at a point $(\theta, \phi)$ can be expressed as

$$
\angle\left(\underline{a}_{\theta}, \underline{a}_{\phi}\right)=\cos ^{-1}\left(\frac{g_{\theta \phi}}{\sqrt{g_{\theta \theta} g_{\phi \phi}}}\right) .
$$

The metric coefficients provide a way for measuring trajectories on the non-Euclidean spaces through the use of weighting coefficients, for example, an infinitesimal distance on the Euclidean plane is measured by $d s^{2}=d x^{2}+d y^{2}$, whereas on a general non-Euclidean surface, it is given by $d s^{2}=g_{\theta \theta} d \theta^{2}+$ $2 g_{\theta \phi} d \theta d \phi+g_{\phi \phi} d \phi^{2}$.

\section{B. Gaussian Curvature}

A surface can be broken down into a set of curves where each curve is described by a set of curvatures. This breakdown is somewhat detrimental to the surface in the sense that each point is described by a curvature related to the specific set of curves rather than the surface itself. By considering the surface as a whole body with intrinsic properties, Gauss assigned a curvature to every point on the surface independently of any specific curve passing through it. Research into surfaces or 2-D manifolds has produced several formulas for the Gaussian curvature originating either from intrinsic properties of the surface, such

\footnotetext{
${ }^{1}$ Note that the tangent plane exists at $\phi= \pm 90^{\circ}$ (which are the apex of the manifold), although the tangent matrix $\mathbb{T}$ has a zero column (coordinate singularity).
}

as the metric, or nonintrinsic properties, such as the observation space. For example, a nonintrinsic formula of the Gaussian curvature exists for surfaces embedded in the 3-D Euclidian space $R^{3 \times 1}$, which involves the normal vector to the surface tangent plane. As the manifold of general array antennas is not embedded in $R^{3 \times 1}$, we will only consider the intrinsic formula for the Gaussian curvature that is independent of the normal space and, hence, of the observation space. An intrinsic formula for the Gaussian curvature $K(\theta, \phi)$ of a surface, adapted from [9], is presented below, expressed as a function of the metric $\mathbb{G}$ as follows:

$$
K(\theta, \phi)=-\frac{1}{\sqrt{\operatorname{det}(\mathbb{G})}}\left(\frac{d \frac{\sqrt{\operatorname{det}(\mathbb{G})}}{g_{\theta \theta}} \Gamma_{\theta \phi}^{\phi}}{d \theta}-\frac{d \frac{\sqrt{\operatorname{det}(\mathbb{G})}}{g_{\theta \theta}} \Gamma_{\theta \theta}^{\phi}}{d \phi}\right)
$$

where $\Gamma_{\theta \phi}^{\phi}, \Gamma_{\theta \theta}^{\phi}$ are elements of the $2 \times 2$ real matrices

$$
\begin{gathered}
\underline{\Gamma}_{2 p} \triangleq \mathbb{G}^{-1} \operatorname{Re}\left\{\mathbb{T}^{H} \frac{d \mathbb{T}}{d p}\right\}=\left[\begin{array}{ll}
\Gamma_{\theta p}^{\theta}, & \Gamma_{\phi p}^{\theta} \\
\Gamma_{\theta p}^{\phi}, & \Gamma_{\phi p}^{\phi}
\end{array}\right] \\
\text { where } p=\theta \text { or } \phi
\end{gathered}
$$

which are known as the Christoffel symbol matrices of the second kind.

The Gaussian curvature of (5) provides an indication of the shape of the surface. For instance, we have the following:

- If $K(\theta, \phi)>0$, the point is elliptic (the whole neighborhood of the surface at the point considered lies on one side of the tangent hyperplane).

- If $K(\theta, \phi)<0$, the point is hyperbolic (one part of the surface at the point considered lies on one side of the tangent hyperplane and the other part on the other side).

- If $K(\theta, \phi)=0$, the point is parabolic (there is a straight line of the surface belonging totally on the tangent space).

- If $K(\theta, \phi)=$ const. $\forall(\theta, \phi)$ then, using Minding's theorem [9], which states that two surfaces of the same constant curvature are locally isometric, we have the following cases. If $K=0$, the surface $\underline{a}(\theta, \phi)$ is isometric with the plane; if $K>0$, the surface $\underline{a}(\theta, \phi)$ is isometric with a sphere of radius $1 / \sqrt{K}$, and if $K<0$, the surface is isometric with a surface of revolution, called a pseudo-sphere, determined by the value $1 / \sqrt{-K}$.

\section{Arc Length}

The arc length $s$ can be interpreted in physical terms as the total distance covered by a person travelling along a certain path on a certain landscape. On the array manifold surface, it is the total distance travelled along a curve on the surface from an "initial" point to the current point under consideration $(\theta, \phi)$. Its expression as a function of the manifold metric is

$$
s=\int_{t} \sqrt{\frac{d \underline{p}^{T}}{d t} \mathbb{G} \frac{d p}{d t}} d t
$$

where $p(t)=[\theta(t), \phi(t)]^{T}$, and hence $d \underline{p} / d t=[d \theta / d t$, $d \phi / d t]^{\bar{T}}$. Note that the expression $I=\|\overline{d \underline{a}}\|^{2}=d \underline{p}^{T} \mathbb{G} d \underline{p}$ 
is a parameter known in differential geometry as the first fundamental form.

\section{Geodesic Curvature}

The final differential geometry parameter of interest presented in this study is the geodesic curvature $\kappa_{g}(s)$. In order to understand the significance of this parameter, the geodesicity concept should be introduced. It is well known that the curve with the minimum length between two points in a Euclidian space is a straight line. This concept is extended to a curve connecting two points on a surface, and the curve of minimum length belonging to the surface is called a geodesic curve (equivalent to a straight line in a plane). By considering a curve on a surface connecting two points, the closeness of this curve to a geodesic curve can be assessed by means of the geodesic curvature $\kappa_{g}$ with $\kappa_{g}=0$ corresponding to a geodesic curve. This curvature can be estimated (for proof, see Appendix A) by the following expression:

$\kappa_{g}(s)=\sqrt{\operatorname{det}(\mathbb{G})}\left[\begin{array}{r}-\frac{d \phi}{d s} \\ \frac{d \theta}{d s}\end{array}\right]^{T}\left\{\left(\underline{\underline{\Gamma}}_{2} \theta \frac{d \theta}{d s}+\underline{\underline{\Gamma}}_{2 \phi} \frac{d \phi}{d s}\right) \frac{d \underline{p}}{d s}+\frac{d^{2} \underline{p}}{d s^{2}}\right\}$

where $s$ is the arc length of the curve under consideration.

From (8), a geodesic curve, i.e., a curve for which the geodesic curvature is invariably zero, must satisfy the equation

$$
\left(\underline{\underline{\Gamma}}_{2} \theta \frac{d \theta}{d s}+\underline{\underline{\Gamma}}_{2 \phi} \frac{d \phi}{d s}\right) \frac{d \underline{p}}{d s}+\frac{d^{2} \underline{p}}{d s^{2}}=\underline{0} \quad \forall(\theta, \phi) .
$$

Equation (9) is a system of two simultaneous equations that are the well-known geodesic differential equations ([9, p. 64]). Note that if two families of curves are orthogonal and one family consists of geodesic curves, then these two families constitute a set of geodesic coordinates.

Using (8), the geodesic curvature $\kappa_{g \theta}$ for $\theta$ curves (i.e., curves of constant elevation parameter $\phi$ ) of an array manifold can be shown, using the properties $d \phi / d s=0$ and $d \theta / d s=1 / \sqrt{g_{\theta \theta}}$, to be equal to

$$
\kappa_{g \theta}=\sqrt{\frac{\operatorname{det}(\mathbb{G})}{g_{\theta \theta}^{3}}} \Gamma_{\theta \theta}^{\phi} .
$$

Similarly, for the $\phi$ curves (i.e., curves of constant azimuth parameter $\theta$ ), using $d \theta / d s=0$ and $d \phi / d s=1 / \sqrt{g_{\phi \phi}}$, the geodesic curvature $\kappa_{g \phi}$ can be found to be

$$
\kappa_{g \phi}=-\sqrt{\frac{\operatorname{det}(\mathbb{G})}{g_{\phi \phi}^{3}}} \Gamma_{\phi \phi}^{\theta} .
$$

It is clear that the computation of both the Gaussian and geodesic curvature parameters [see (5) and (8)] involves the elements of the matrices $\underline{\underline{\Gamma}}_{2 \theta}$ and $\underline{\underline{\Gamma}}_{2 \phi}$. These elements are known as the Christoffel symbols of the second kind, having the property $\Gamma_{\theta \phi}^{p}=\Gamma_{\phi \theta}^{p}$, and are used as a transformation that provides the infinitesimal variation of the tangent plane as a point moves on the manifold surface, namely

$$
d \mathbb{}=\mathbb{\mathbb { T }} \underline{\underline{\Gamma}}=\mathbb{T}\left(\underline{\underline{\Gamma}}_{2 \theta} d \theta+\underline{\underline{\Gamma}}_{2 \phi} d \phi\right) .
$$

Furthermore, note that $\underline{\Gamma}_{2 \theta}$ and $\underline{\Gamma}_{2 \phi}$ are related to the Christoffel symbol matrices of the first kind (not used in this study) $\underline{\underline{\Gamma}}_{1 \theta}$ and $\underline{\underline{\Gamma}}_{1 \phi}$ as follows:

$$
\underline{\underline{\Gamma}}_{2 p}=\mathbb{G}^{-1} \underline{\underline{\Gamma}}_{1 p} \text { where } p=\theta \text { or } \phi .
$$

The previously described parameters will be employed in the following section to represent the array manifold surface, embedded in a complex multidimensional space, as a real parameter plane, while preserving its main characteristics. This has the potential to simplify the analysis of the manifold or any future algorithmic design.

\section{Proposed Representation of the Manifold Surface}

The main idea is to propose a local mapping to represent a complicated surface (embedded in a multidimensional complex space) by a simpler surface. This representation would simplify the analysis of array manifold surfaces and, consequently, of array systems. The simplest surface, from a conceptual point of view, is the real plane, and hence, in this section, we will propose a mapping to transform the manifold surface onto a real plane. In order to be useful, this mapping should preserve the main characteristics of the manifold surface. These are, for instance, the Gaussian curvature, which may or may not be constant, and the geodesic properties. Based on the fact that a real 2-D plane has a constant Gaussian curvature (equal to zero) and straight geodesic lines, some constraints on our mapping can be established. A manifold surface with a varying Gaussian curvature cannot be adequately mapped onto the plane. In addition, the transformation should map geodesic parameter curves on the manifold surface onto straight lines on the real plane that are the geodesic curves of the plane (geodesic mapping). Based on the above discussion, a mapping is proposed below in the form of a theorem.

Theorem 1: Consider an arbitrary point $(\theta, \phi)$ on the manifold surface of an array of $N$ sensors. The image of the point $(\theta, \phi)$ on the real parameter plane is given by

$$
\underline{D}(\theta, \phi)=\left[\begin{array}{c}
x_{0}(\phi)+\int_{0}^{\theta} \kappa_{g \theta}^{-1}(\vartheta, \phi) \cos (\vartheta) d \vartheta \\
y_{0}(\phi)+\int_{0}^{\theta} \kappa_{g \theta}^{-1}(\vartheta, \phi) \sin (\vartheta) d \vartheta
\end{array}\right]
$$

where $\kappa_{g \theta}(\theta, \phi)$ is the geodesic curvature of the $\theta$ curves on the manifold, and $x_{0}(\phi)$ and $y_{0}(\phi) \in R$ are integration constants such that

and

$$
x_{0}=-\frac{1}{2} \int_{0}^{\pi} \kappa_{g \theta}^{-1}(\vartheta, \phi) \cos (\vartheta) d \vartheta
$$

$$
y_{0}=-\frac{1}{2} \int_{0}^{\pi} \kappa_{g \theta}^{-1}(\vartheta, \phi) \sin (\vartheta) d \vartheta
$$

The locus of the images $\underline{D}(\theta, \phi) \forall(\theta, \phi)$ is known as the development ${ }^{2}$ of the manifold and has the following properties:

i) It exists if and only if the Gaussian curvature of the manifold surface is constant.

${ }^{2}$ More on "development" and "developables" can be found in [12]. 
ii) The arc length $s_{d \theta}\left(\theta, \phi_{0}\right)$ of $\theta$ curves on the development is given by

$$
s_{d \theta}\left(\theta, \phi_{0}\right)=\int_{\theta} \kappa_{g \theta}^{-1}\left(\vartheta, \phi_{0}\right) d \vartheta .
$$

iii) The curvature of the $\theta$ curves on the development is equal to the geodesic curvature $\kappa_{g \theta}\left(\theta, \phi_{0}\right)$ of the $\theta$ curves on the manifold surface.

Proof:

i) Beltrami's theorem [13], [14] states that if a surface is mapped to another surface and the mapping is such that geodesic curves in one surface are mapped onto geodesic curves in the other surface (geodesic mapping) then if one surface has a constant Gaussian curvature, the other must also have a constant Gaussian curvature in order to have a distortionless mapping. Since the destination surface of our mapping, i.e., the plane, has a constant curvature (equal to zero), the condition for the existence of a distortionless mapping is that the array manifold surface should have a constant curvature.

ii) Considering the development of the manifold $\theta$ curves for a constant $\phi_{0}, \underline{D}_{\theta}(\theta)=\underline{D}\left(\theta, \phi_{0}\right) \forall \theta$, the arc length of $\underline{D}_{\theta}(\theta)$ is given by

$$
s_{d \theta}(\theta)=\int_{\theta}\left\|\frac{d \underline{D}_{\theta}(\vartheta)}{d \vartheta}\right\| d \vartheta .
$$

Using the Leibnitz rule of integral differentiation on (14)

$$
\frac{d \underline{D}_{\theta}(\theta)}{d \theta}=\kappa_{g \theta}^{-1}\left(\theta, \phi_{0}\right)\left[\begin{array}{l}
\cos (\theta) \\
\sin (\theta)
\end{array}\right]
$$

and hence $\left\|d \underline{D}_{\theta}(\theta) / d \theta\right\|=1 / \kappa_{g \theta}\left(\theta, \phi_{0}\right)$, proving (15).

iii) The curvature of a curve is the magnitude of the derivative of the normalized tangent vector with respect to its arc length hence, considering the development of the manifold $\theta$ curves for a constant $\phi_{0}$, let $\underline{u}(\theta)$ be its normalized tangent vector

$$
\underline{u}(\theta)=\frac{d \underline{D}_{\theta}(\theta)}{d \theta} /\left\|\frac{d \underline{D}_{\theta}(\theta)}{d \theta}\right\|= \pm\left[\begin{array}{c}
\cos (\theta) \\
\sin (\theta)
\end{array}\right] .
$$

Differentiating (15) leads to $d s_{d \theta}=d \theta / \kappa_{g \theta}\left(\theta, \phi_{0}\right)$, and hence, $d \theta / d s_{d \theta}=\kappa_{g \theta}\left(\theta, \phi_{0}\right)$.

The magnitude of the derivative of the normalized tangent vector with respect to its arc length hence becomes

$$
\begin{aligned}
\left\|\frac{d \underline{u}(\theta)}{d s_{d \theta}}\right\|=\left\|\frac{d \underline{u}(\theta)}{d \theta} \frac{d \theta}{d s_{d \theta}}\right\| & =\left\|\left[\begin{array}{c}
-\sin (\theta) \\
\cos (\theta)
\end{array}\right] \kappa_{g \theta}\left(\theta, \phi_{0}\right)\right\| \\
& =\kappa_{g \theta}\left(\theta, \phi_{0}\right) .
\end{aligned}
$$

The curvature of the development is thus equal to the geodesic curvature of the manifold curve.

Note that the expressions for the integration constants assume the property $\kappa_{g \theta}(\theta, \phi)=\kappa_{g \theta}(\theta+\pi, \phi)$ (which is generally valid) and have the effect of shifting the curves to a common center, i.e., $\underline{D}(\theta, \phi)+\underline{D}(\theta+\pi, \phi)=\underline{0}$.

The motivation behind the conservation of the geodesic curvatures is to maintain the characteristic that the shortest path between two points on the surface maps to a straight line on the plane (i.e., the shortest path on the development); otherwise, the development will represent a "distorted" surface. Furthermore, the geodesic curves on the array manifold have been investigated (e.g., see [8]) and have proved to be "hyperhelices" (constant curvatures) embedded in an $N$-dimensional complex space. The advantages of having hyperhelical curves are numerous. The most important is that their shape and properties can be described by a set of constant curvatures that can be analytically estimated. Note that curves of constant azimuth and varying elevation ( $\phi$ curves) are of this type and are expected to map to straight lines on the development, whereas $\theta$ curves, which are curves of constant elevation, are not. Another parameterization of the array manifold (not referred to in this paper) is the $(\alpha, \beta)$ parameterization [15], where both $\alpha$ curves and $\beta$ curves are hyperhelices, and both are mapped to straight lines on the development.

\section{ApPlicAtion of DifFERENTIAL GeOMETRY to ARRAYS WITH OMNIDIRECTIONAL SENSORS}

In order to demonstrate the feasibility and potential benefit of the differential geometry parameters defined above and the proposed concept of the development, in this section, a general array of omnidirectional sensors will be considered. This implies that the manifold vector given by (1) is simplified to

$$
\underline{a}(\theta, \phi)=\exp \left(-j \underline{\underline{r}}^{T} \underline{k}\right) .
$$

In addition, the first and second derivatives of the manifold vector are essential for evaluating these parameters, and hence, the following notation will be used:

$\underline{k}_{p}=\frac{d \underline{k}}{d p} \quad$ and $\quad \underline{k}_{p q}=\frac{d^{2} \underline{k}}{d p d q} \quad$ where $(p$ or $q)=(\theta$ or $\phi)$.

Thus, for arrays of omnidirectional sensors, the elements of the metric $\mathbb{G}$ can be computed as

$$
g_{p q}=\underline{k}_{p}^{T} \underline{\underline{r}}_{\underline{\underline{r}}}^{T} \underline{k}_{q} \quad \text { where } p \text { and } q=\theta \text { or } \phi
$$

whereas the Christoffel symbol matrices of the second kind are

$$
\underline{\underline{\Gamma}}_{2 \theta}=\left[\begin{array}{cc}
\Gamma_{\theta \theta}^{\theta}, & -\tan \phi \\
\Gamma_{\theta \theta}^{\phi}, & 0
\end{array}\right]
$$

and

$$
\underline{\underline{\Gamma_{2}}}=\left[\begin{array}{cc}
-\tan \phi, & \Gamma_{\phi \phi}^{\theta} \\
0, & \Gamma_{\phi \phi}^{\phi}
\end{array}\right] \text {. }
$$

It is clear from (18) that $\Gamma_{\theta \phi}^{\phi}=0$, and this simplifies, for omnidirectional sensors, the Gaussian curvature of (5) as follows:

$$
K(\theta, \phi)=\frac{1}{\sqrt{\operatorname{det}(\mathbb{G})}} \frac{d}{d \phi}\left(\frac{\sqrt{\operatorname{det}(\mathbb{G})}}{g_{\theta \theta}} \Gamma_{\theta \theta}^{\phi}\right)
$$

which, in general, is not constant. Thus, the existence of the development of the manifold of an array of omnidirectional sensors is not guaranteed according to Part-i of the theorem. However, for some array geometries, the Gaussian curvature of (19) is constant. Array geometries that have been identified to satisfy this condition include all planar arrays and some 3-D arrays. In 


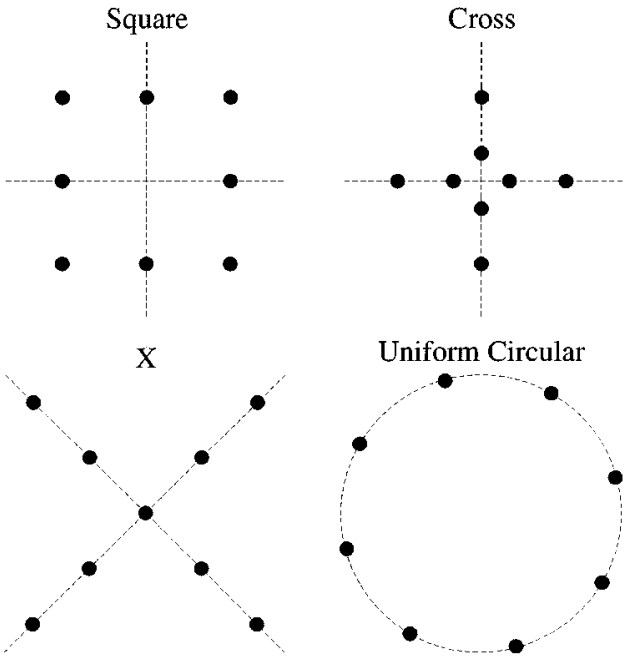

Fig. 1. Various 2-D-grid array geometries.

TABLE I

ANALYTICAL EXPRESSIONS OF DIFFERENTIAL GEOMETRY PARAMETERS FOR DEVELOPABLE ARRAYS OF OMNIDIRECTIONAL SENSORS

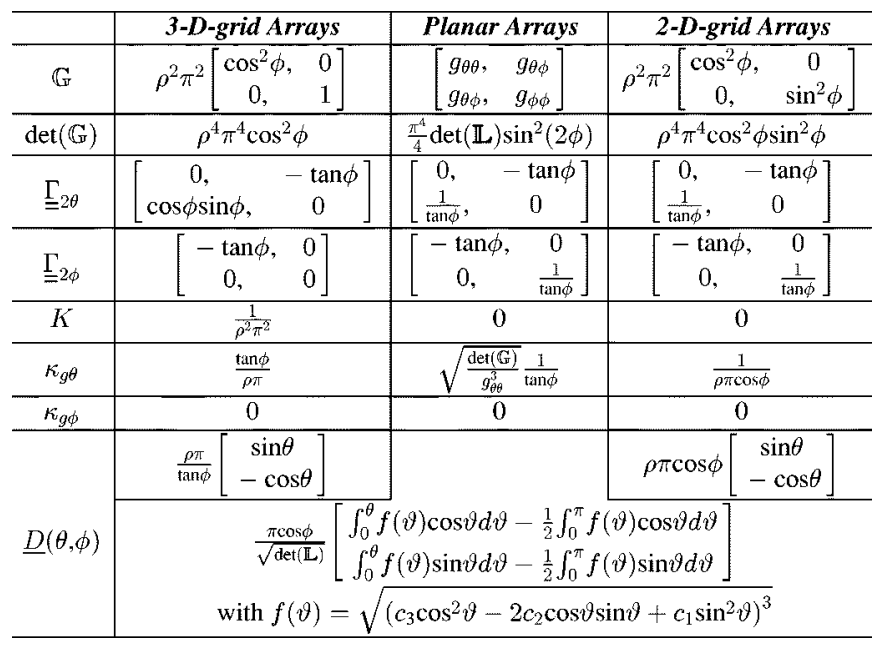

general, a 3-D array is not "developable," but the special class of 3-D-grid arrays defined below is developable.

Definition 1-3-D-Grid Arrays: A 3-D array geometry of omnidirectional sensors is said to be a 3-D-grid array if and only if the following expression is satisfied:

$$
\underline{\underline{r}} \underline{\underline{r}}^{T}=\rho^{2} \rrbracket_{3} \text { where } \rho \in R
$$

with $\mathbb{1}_{n}$ denoting an $n \times n$ identity matrix.

An example of a 3-D-grid array is the eight-element cube array with all sides equal to one half wavelength.

Note that for planar arrays, the term $\underline{\underline{r}} \underline{\underline{r}}^{T}$ has the form $\left[\begin{array}{cc}\mathbb{L}, & \underline{0} \\ \underline{0}^{T} & 0\end{array}\right]$, where $\mathbb{L}=\left[\begin{array}{cc}c_{1}, & c_{2} \\ c_{2}, & c_{3}\end{array}\right] \in R^{2 \times 2}$, and if $\mathbb{L}=\rho^{2} \rrbracket_{2}$ with $\rho \in R$, then the planar array will be known as a 2-D-grid array. Fig. 1 shows some examples of 2-D-grid array geometries.

Table I summarizes the results of the evaluation of the differential geometry parameters introduced in this study for three classes of arrays of omnidirectional sensors. These are the 3-Dgrid, planar and 2-D-grid arrays. A number of comments can be made based on the results presented in Table I. The Gaussian curvature of 3-D-grid arrays is always positive, whereas for

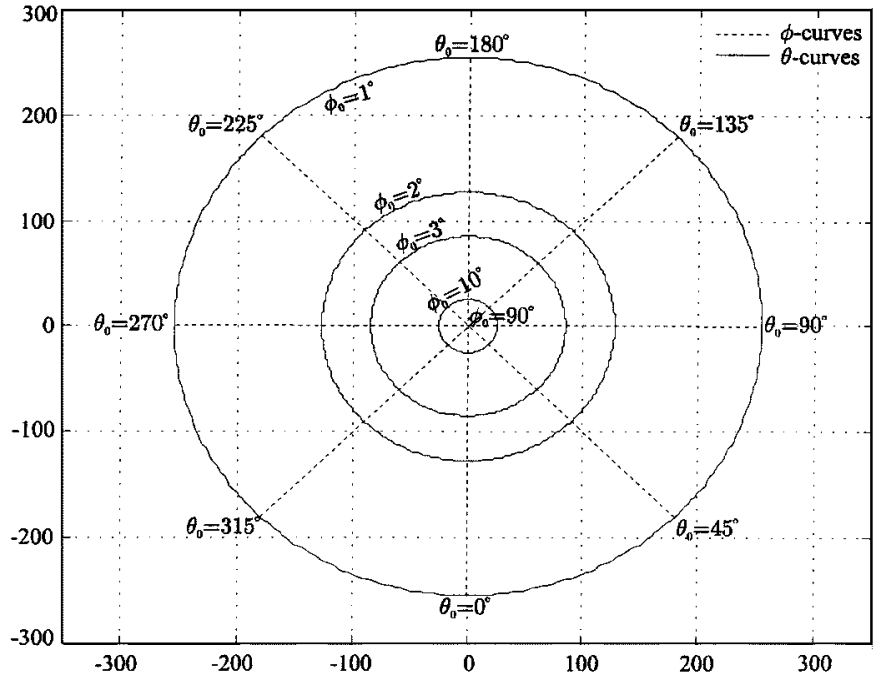

Fig. 2. $\theta$ curves and $\phi$ curves development of the manifold of a 3-D-grid array with sensors located on the eight vertices of a cube of side one half-wavelength and with reference point the center of the cube (array centroid).

planar arrays, it is always zero (for proof, see Appendix B). This implies that the manifold of a 3-D-grid array is isometric with a sphere of radius $\rho \pi$ and that of a planar array is isometric with a plane. In addition, it can be seen that all these arrays have a zero geodesic curvature for the $\phi$ curves, which implies that the $\phi$ curves are geodesic curves. Furthermore, for the 2-D-grid and 3-D-grid arrays, the off-diagonal elements of the manifold metric $\mathbb{G}$ (i.e., $g_{\theta \phi}, g_{\phi \theta}$ ) are zero, and hence, the $\theta$ curves and $\phi$ curves are orthogonal and constitute geodesic coordinates, whereas the development of their $\theta$ curves are circles whose radius is a function of $\phi_{0}$. Finally, for planar arrays, the expression presented in Table I (for proof, see Appendix C) indicates that the determinant of the manifold metric $\mathbb{G}$ is independent of the parameter $\theta$.

Figs. 2-4 provide the development of representative 3-D-grid, planar, and 2-D-grid array geometries. For the planar array of Fig. 3, the positions of the sensors are

$$
\underline{\underline{r}}=\left[\begin{array}{ccccc}
-2.1 & -1.1 & 0.4 & 0.9 & 1.9 \\
-2.4 & 1.1 & 0.6 & -0.4 & 1.1 \\
0 & 0 & 0 & 0 & 0
\end{array}\right]
$$

in half wavelengths.

It can be seen from Fig. 3 that the development of the planar array has an ellipsoidal shape with the two main axes at angles $\psi_{0}$ and $\psi_{0}+\pi / 2$ with respect to the $x$ axis, where

$$
\psi_{0}=\frac{1}{2} \tan ^{-1}\left(\frac{2 c_{2}}{c_{1}-c_{3}}\right)
$$

with $c_{i}$ denoting the elements of the matrix $\mathbb{L}$, i.e., $\mathbb{L}=\left[\begin{array}{ll}c_{1}, & c_{2} \\ c_{2}, & c_{3}\end{array}\right]$. The proof of this result can be found in Appendix D.

\section{Potential ApPlications}

In this section, an attempt is made to reveal a few potential applications of the differential geometry tools described in this paper and, thus, to demonstrate their benefits in the area of array processing. 


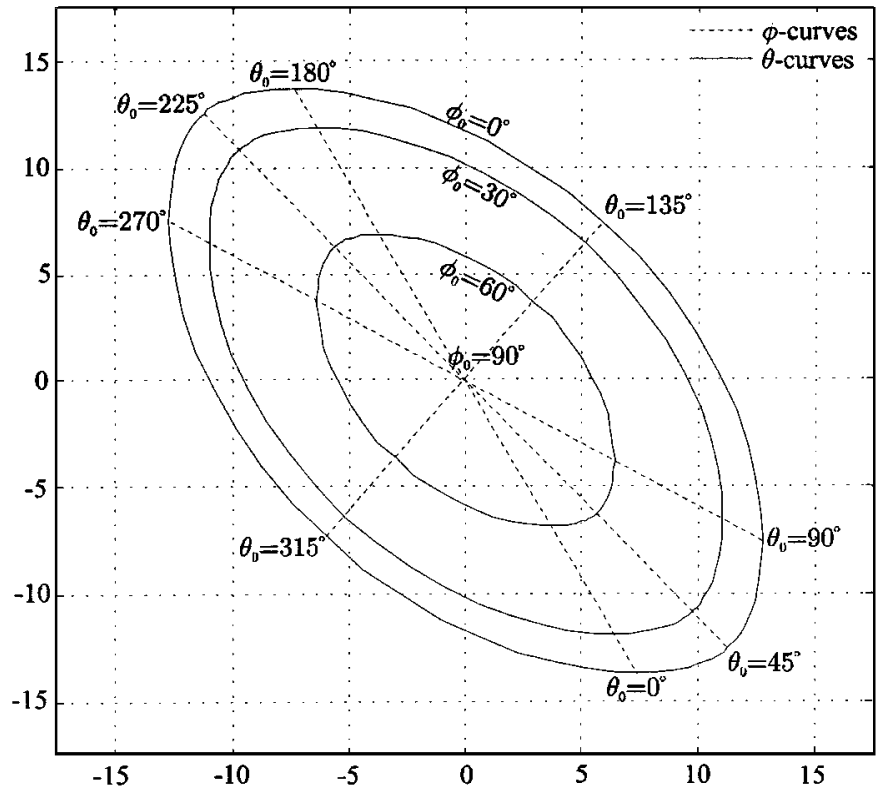

Fig. 3. $\theta$ curves and $\phi$ curves development of the manifold of a planar array of five-sensor with positions given by (21).

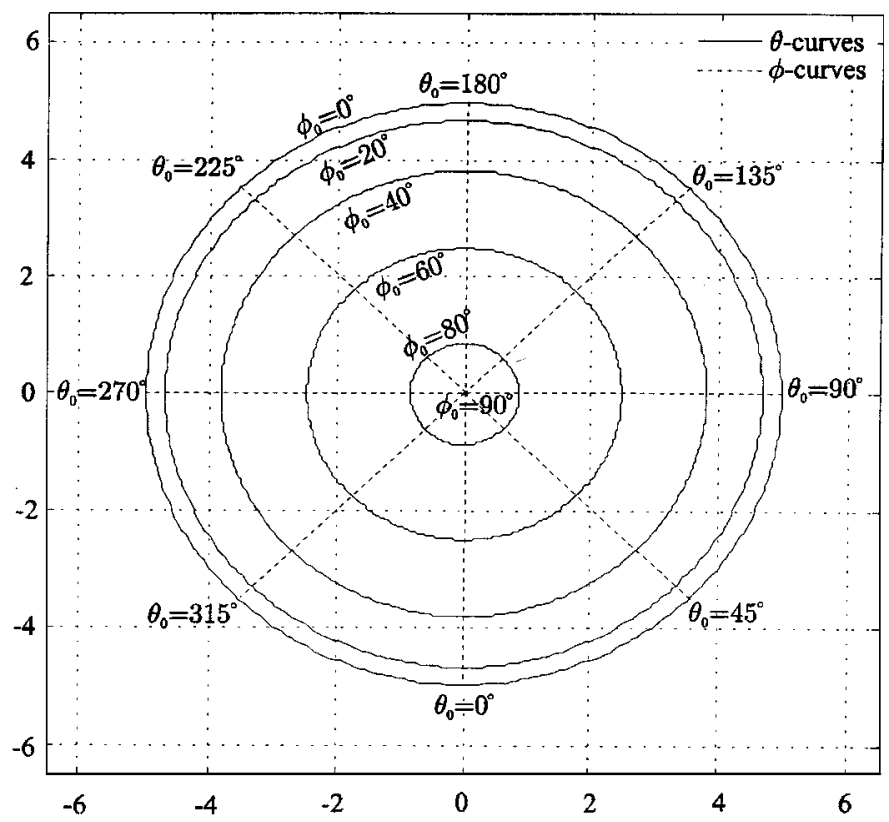

Fig. 4. $\theta$ curves and $\phi$ curves development of the manifold of a five-sensor uniform circular array (2-D-grid array) of radius 1 half wavelength.

\section{A. Algorithm Design Based on Shape of the Manifold Surface}

The significance of the shape of the manifold surface in a direction-finding array system becomes apparent when it is noted that the manifold surface has to be searched, according to a "cost" function in order to estimate some parameters of interest (e.g., directions of arrival). This cost function can be designed and its "search" optimized according to the shape of the manifold surface and its properties. A similar approach for curvilinear manifolds, rather than surfaces, can be found in [16]. Based on the concepts presented in this paper, it can be easily proven that the shape of the manifold of an array of omnidirec-

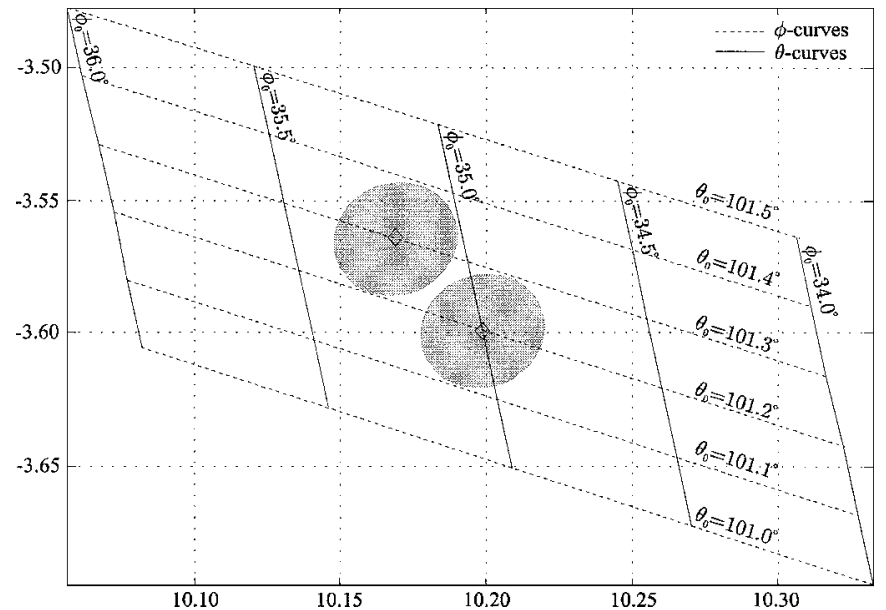

Fig. 5. Development of uncertainty spheres around the bearings of two sources at $\left(101.2^{\circ}, 35^{\circ}\right)$ and $\left(101.3^{\circ}, 35.2^{\circ}\right)$ for the array of (21) corresponding to an $\mathrm{SNR}=20 \mathrm{~dB}$ and $L=32$ snapshots.

tional sensors is a conoid, as highlighted by the following theorem.

Theorem 2: The manifold surface of a planar array of omnidirectional sensors is a conoid embedded in a complex multidimensional space.

A proof of this theorem can be found in [17]. From the results of this study, we believe that new cost functions may be proposed that take into account the conoidal shape of the manifold in their design and incorporate various differential geometry array manifold parameters.

\section{B. Array Design Through Differential Geometry Manifold Parameters}

An important issue that has, however, been overlooked is the design of the array structure (geometry) that, under practical nonasymptotic conditions, places fundamental limitations on the ultimate performance of the system. A potential application is to propose a new array design framework based on the differential geometry array manifold parameters. For instance, the sensor locations of a planar array are related to the metric $\mathbb{G}$ and to two geodesic curves ( $\phi$ curves at $\left.\theta_{1}, \theta_{2}\right)$, as can be seen in the following theorem, which is presented without any proof.

Theorem 3: The $x$ and $y$ coordinates of the elements of a planar array of $N$ sensors are related to the differential geometry parameters of its manifold surface as follows:

$$
\begin{aligned}
{\left[\underline{r}_{\boldsymbol{x}}, \underline{r}_{\boldsymbol{y}}\right]=} & \frac{1}{\pi \sin \left(\theta_{2}-\theta_{1}\right)}\left[\underline{\rho}_{\theta_{1}}, \underline{\rho}_{\theta_{2}}\right] \\
& \cdot\left[\begin{array}{rr}
\sqrt{g_{\phi \phi}\left(\theta_{1}, 90\right)} \sin \theta_{2}, & -\sqrt{g_{\phi \phi}\left(\theta_{1}, 90\right)} \cos \theta_{2} \\
-\sqrt{g_{\phi \phi}\left(\theta_{2}, 90\right)} \sin \theta_{1}, & \sqrt{g_{\phi \phi}\left(\theta_{2}, 90\right)} \cos \theta_{1}
\end{array}\right]
\end{aligned}
$$

where $\theta_{1}, \theta_{2}$ are any two known azimuths satisfying $\theta_{1}-\theta_{2} \neq$ $0 \bmod \pi$, and $\underline{\rho}_{\theta}$ is the vector of the $N$ roots of the sensor locator polynomial ${ }^{3}$

$$
p\left(\tilde{r}_{\theta}\right)=\tilde{r}_{\theta}^{d}-b_{2}(\theta) \tilde{r}_{\theta}^{d-2}+b_{3}(\theta) \tilde{r}_{\theta}^{d-4}-\cdots+b_{(d / 2)+1}(\theta) .
$$

${ }^{3} \mathrm{~A}$ full investigation of the sensor locator polynomial and the synthesis of Theorem 3 can be found in [18]. 


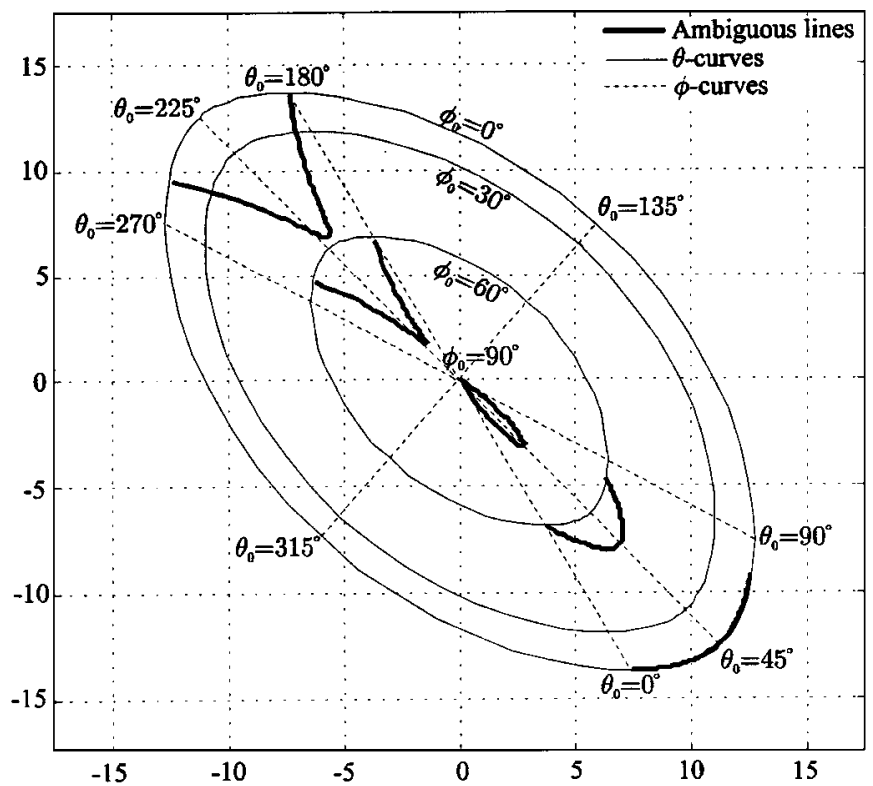

Fig. 6. Development of the array manifold for the array of (21). The solid lines represent the mapping of a set of ambiguous generator lines.

It is important to note that the entries of $\underline{\rho}_{\theta_{1}}$ and $\underline{\rho}_{\theta_{2}}$ can be taken in every possible order. Indeed, because each entry of $\rho_{\theta_{1}}$ can be set to correspond to an arbitrary entry of $\rho_{\theta_{2}}$, the previous theorem gives a whole family of planar arrays whose manifolds have the specified differential geometry.

\section{Performance Bounds Based on the Concept of Development}

The effect of the array structure on the system performance may be assessed quantitatively by determining the shape and orientation of the array manifold through the study of the manifold's differential geometry. Estimation error, detection, and resolution bounds have been established as a function of the differential geometry parameters for the case of linear arrays where the array manifold is a curve having the shape of a hyperhelix embedded in a multidimensional complex space [1]-[3]. These bounds are based on the concept of "uncertainty spheres" that model the uncertainty remaining in the system after $L$ snapshots. A generalization of this method for manifold surfaces is not straightforward but may be handled by using the concept of the development proposed in this study to map these uncertainty spheres onto the real parameter plane. An example of this mapping can be seen in Fig. 5. However, further investigation is necessary to demonstrate the potential impact of this idea on detection and resolution bounds.

\section{Investigation of Ambiguities Based on the Array Manifold Development}

The geometry of an array plays a crucial role in dictating the shape, properties and "anomalies" of the array manifold and, as a consequence, in dictating the phenomenon where some manifold vectors can be written as linear combinations of others. This gives rise to the array "ambiguity problem" [4], [5], which is an important research topic. This is a promising potential application area of the proposed mapping because it provides a way of visualizing ambiguous sets of directions of arrival and henceforth ambiguous regions, thereby distinguishing geometries that are more (or less) ambiguous than others. As an example, Fig. 6 illustrates a set of ambiguous generator lines [5] for a given planar array mapped on the development of the manifold. In this figure, the intersection between any line through the apex $\left(\phi=90^{\circ}\right)$ and the solid lines provides a set of five ambiguous directions. This representation may lead to a better understanding of the ambiguity problem with implications for the identification of new classes of ambiguities and the design of ambiguity-free (up to a certain rank) array geometries.

\section{CONCLUSIONS}

In this paper, the main differential geometry parameters of the manifold surface of a nonlinear array of sensors have been identified, and analytical expressions have been provided. In addition, the concept of the development has been proposed, under certain conditions, as an alternative representation of the manifold surface on a real plane, maintaining its main differential geometry characteristics. The proposed concepts have been applied to 3-D and planar arrays of omnidirectional sensors, and a number of potential applications have been discussed.

\section{APPENDIX}

\section{A. Proof of (8)-Geodesic Curvature}

The geodesic curvature $\kappa_{g}$ is explicitly derived in this Appendix as a function of the parameters presented in Section II. Consider the manifold surface of an array that is the locus of the manifold vectors $\underline{a}(\theta, \phi) \forall(\theta, \phi)$. Let us define the tangent vector at a point $(\theta, \phi)$ of a curve on the surface as

$$
\underline{a}_{s} \equiv \frac{d \underline{a}(s)}{d s}=\mathbb{T} \frac{d \underline{p}}{d s}
$$

where $s$ is the arc length of the curve, and T is defined in Section II as $\mathbb{T}=\left[\underline{a}_{\theta}, \underline{a}_{\phi}\right] \in C^{N \times 2}$ such that $\mathcal{L}\{\mathbb{T}\}$ is the tangent space to the manifold surface at $(\theta, \phi)$ and $\underline{p}(s)=[\theta(s), \phi(s)]^{T}$. Note that $\mathcal{L}\{$ matrix $\}$ denotes the space spanned by the columns of the matrix argument. Furthermore, let us define the matrix $\mathbb{B}=\left[\underline{b}_{1}, \underline{b}_{2}\right] \in C^{N \times 2}$ as an orthonormal basis for the space $\mathcal{L}\{\mathbb{T}\}$ with $\underline{b}_{1}$ chosen to be equal to $\underline{a}_{s} /\left\|\underline{a}_{s}\right\|$. This gives the expression

$$
\mathbb{B}=\left[\underline{b}_{1}, \underline{b}_{2}\right]=\mathbb{\mathbb { E }} \mathbb{E}_{\theta} \mathbb{H}
$$

where

$$
\mathbb{E}_{\theta}=\frac{1}{\sqrt{\operatorname{det}(\mathbb{G})}}\left[\begin{array}{cc}
\sqrt{\frac{\operatorname{det}(\mathbb{G})}{g_{\theta \theta}}}, & -\frac{g_{\theta \phi}}{\sqrt{g_{\theta \theta}}} \\
0, & \sqrt{g_{\theta \theta}}
\end{array}\right]
$$

is the Gram-Schmidt orthonormalization matrix $\mathbb{E}_{\theta} \in R^{2 \times 2}$ such that the first column of $\mathbb{T} \mathbb{E}_{\theta}$ is the basis vector $\underline{a}_{\theta} /\left\|\underline{a}_{\theta}\right\|$

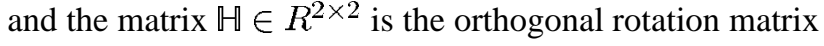

$$
\mathbb{H}=\left[\begin{array}{rr}
\cos \psi, & -\sin \psi \\
\sin \psi, & \cos \psi
\end{array}\right]
$$


where $\psi$ is the rotation angle between the tangent vector to the $\theta$ curve $\underline{a}_{\theta}$ and the tangent vector to the curve under consideration $\underline{a}_{s}$

$$
\psi=\angle\left(\underline{a}_{\theta}, \underline{a}_{s}\right)=\cos ^{-1}\left(\frac{\operatorname{Re}\left\{\underline{a}_{\theta}^{H} \underline{a}_{s}\right\}}{\left\|\underline{a}_{\theta}\right\|\left\|\underline{a}_{s}\right\|}\right) .
$$

By projecting the second derivative $\underline{a}_{s s} \equiv d \underline{a}_{s} / d s=$ $(d \pi / d s)(d p / d s)+\mathbb{T}\left(d^{2} p / d s^{2}\right)$ of the manifold vector at $(\theta, \phi)$ onto the subspace $\mathcal{L}\{\mathbb{B}\}(\mathcal{L}\{\mathbb{B}\}=\mathcal{L}\{\mathbb{T}\})$, we have

$$
\underline{\underline{P}}_{\mathbb{R}} \underline{\underline{a}}_{S s}=\kappa_{1} \underline{b}_{1}+\kappa_{g} \underline{b}_{2}
$$

where $\underline{\underline{P}}_{\mathbb{B}}=\mathbb{B}\left(\mathbb{B}^{H} \mathbb{B}\right)^{-1} \mathbb{B}^{H}$ is the projection operator. The coefficient of $\underline{b}_{2}$, that is $\kappa_{g}$, is defined as the geodesic curvature of the curve at $(\theta, \phi)$ and represents the component of $\underline{a}_{s s}$ in the direction of $\underline{b}_{2}$. Hence, its expression is

$$
\kappa_{g}=\operatorname{Re}\left\{{\underline{b_{2}}}^{H} \underline{a}_{s s}\right\} .
$$

The vector $\underline{b}=[0,1]^{T}$ will be used for notational purposes such that $\underline{b}_{2}=\mathbb{B}$.

Defining a general curve on the $(\theta, \phi)$ parameter plane $\underline{p}=$ $p(s)=[\theta(s), \phi(s)]^{T}$ as a function of the manifold curve $\operatorname{arc}$ length, the following results follow.

Substituting the expressions of $\underline{b}_{2}$ and $\underline{a}_{s s}$ into (28) gives, after some manipulation

$$
\kappa_{g}(s)=\underline{b}^{T} \mathbb{T}^{T} \mathbb{E}_{\theta}^{T} \mathbb{G}\left\{\left(\underline{\underline{\Gamma}} 2 \theta \frac{d \theta}{d s}+\underline{\underline{\Gamma_{2}}} 2 \frac{d \phi}{d s}\right) \frac{d \underline{p}}{d s}+\frac{d^{2} \underline{p}}{d s^{2}}\right\} .
$$

From (29), the term $\underline{b}^{T} \mathbb{\boxplus}^{T} \mathbb{E}_{\theta}^{T} \mathbb{G}$ can be simplified and gives, after some manipulation

$$
\begin{aligned}
\kappa_{g}(s)= & \sqrt{\operatorname{det}(\mathbb{G})}\left[\begin{array}{r}
-\frac{d \phi}{d s} \\
\frac{d \theta}{d s}
\end{array}\right]^{T} \\
& \cdot\left\{\left(\underline{\Gamma}_{2} 2 \frac{d \theta}{d s}+\underline{\underline{\Gamma}}_{2 \phi} \frac{d \phi}{d s}\right) \frac{d \underline{p}}{d s}+\frac{d^{2} \underline{p}}{d s^{2}}\right\} .
\end{aligned}
$$

\section{B. Proof that the Gaussian Curvature of an Omnidirectional Sensor Planar Array Manifold is Zero}

Equation (19) gives the expression of the Gaussian curvature of an array of omnidirectional sensors that can be written in an equivalent way as in (31), shown at the bottom of the page. By using the Christoffel symbols of the second kind, $\Gamma_{\theta \phi}^{\theta}$ and $\Gamma_{\phi \phi}^{\phi}$, which can be expressed in terms of the element of the manifold metric $\mathbb{G}$ as

$$
\left\{\begin{array}{l}
\Gamma_{\theta \phi}^{\theta}=\frac{1}{2 \operatorname{det}(\mathbb{G})}\left(g_{\phi \phi} \frac{d g_{\theta \theta}}{d \phi}-g_{\theta \phi} \frac{d g_{\phi \phi}}{d \phi}\right) \\
\Gamma_{\phi \phi}^{\phi}=\frac{1}{2 \operatorname{det}(\mathbb{G})}\left(g_{\theta \theta} \frac{d g_{\phi \phi}}{d \phi}+g_{\theta \phi} \frac{d g_{\phi \phi}}{d \phi}-2 g_{\theta \phi} \frac{d g_{\theta \phi}}{d \phi}\right) .
\end{array}\right.
$$

then (31) can be written as

$$
K=\frac{\Gamma_{\theta \phi}^{\theta}+\Gamma_{\phi \phi}^{\phi}}{g_{\theta \theta} \tan \phi}-\frac{\frac{d g_{\theta \theta}}{d \phi} \tan \phi+g_{\theta \theta}\left(1+\tan ^{2} \phi\right)}{g_{\theta \theta}^{2} \tan ^{2} \phi} .
$$

Using the previous expression in conjunction with Table I, which provides the Christoffel symbols $\Gamma_{\theta \phi}^{\theta}$ and $\Gamma_{\phi \phi}^{\phi}$ for a planar array as a function of $\phi$, we have

$$
\begin{aligned}
K & =\frac{-\tan \phi+\frac{1}{\tan \phi}}{g_{\theta \theta} \tan \phi}-\frac{\frac{d g_{\theta \theta}}{d \phi} \tan \phi+g_{\theta \theta}\left(1+\tan ^{2} \phi\right)}{g_{\theta \theta}^{2} \tan ^{2} \phi} \\
& =-\frac{\frac{d g_{\theta \theta}}{d \phi}+2 g_{\theta \theta} \tan \phi}{g_{\theta \theta}^{2} \tan \phi} .
\end{aligned}
$$

By using the properties

$$
\left\{\begin{array}{l}
\frac{d g_{\theta \theta}}{d \phi}=\frac{d \underline{k}_{\theta}^{T} \underline{\underline{r}}^{\underline{r}} \underline{\underline{r}}^{T} \underline{k}_{\theta}}{d \phi}=2 \underline{k}_{\theta}^{T} \underline{\underline{r}}_{\underline{\underline{r}}}^{\underline{\underline{r}}} \underline{\underline{k}}_{\theta \phi} \\
\underline{k}_{\theta \phi}=-\underline{k}_{\theta} \tan \phi
\end{array}\right.
$$

we have $K=-\left(-2 g_{\theta \theta} \tan \phi+2 g_{\theta \theta} \tan \phi\right) /\left(g_{\theta \theta}^{2} \tan \phi\right)=0$.

\section{Proof of the Expression of $\operatorname{det}(\mathbb{G})$ for Planar Arrays in} Table I

The determinant of the manifold metric $\mathbb{G}$ can be easily proven for grid arrays (see first and third columns of Table I); therefore, in this Appendix, we will only consider the proof for planar arrays (second column). For planar arrays, the term $\underline{\underline{r}} \underline{\underline{r}}^{T}$ has the form $\left[\begin{array}{cc}\mathbb{L}, & 0 \\ 0, & 0\end{array}\right]$, where $\mathbb{L}=\left[\begin{array}{cc}c_{1}, & c_{2} \\ c_{2}, & c_{3}\end{array}\right] \in R^{2 \times 2}$, and the elements of the manifold metric $\mathbb{G}$ for an array of omnidirectional sensors can be explicitly written as a function of the elements of $\mathbb{L}$ as

$$
\left\{\begin{array}{c}
g_{\theta \theta}=\pi^{2} \cos ^{2} \phi\left(c_{3} \cos ^{2} \theta-2 c_{2} \cos \theta \sin \theta+c_{1} \sin ^{2} \theta\right) \\
g_{\phi \phi}=\pi^{2} \sin ^{2} \phi\left(c_{1} \cos ^{2} \theta+2 c_{2} \cos \theta \sin \theta+c_{3} \sin ^{2} \theta\right) \\
g_{\theta \phi}=\pi^{2} \cos \phi \sin \phi\left(c_{2}\left(\sin ^{2} \theta-\cos ^{2} \theta\right)\right. \\
\left.+\left(c_{1}-c_{3}\right) \cos \theta \sin \theta\right) .
\end{array}\right.
$$

$$
\begin{aligned}
K & =\frac{1}{\sqrt{\operatorname{det}(\mathbb{G})}} \frac{\frac{1}{2}\left(g_{\theta \theta} \frac{d g_{\phi \phi}}{d \phi}+g_{\phi \phi} \frac{d g_{\theta \theta}}{d \phi}-2 g_{\theta \phi} \frac{d g_{\theta \phi}}{d \phi}\right) \sqrt{\operatorname{det}(\mathbb{G})}-1 g_{\theta \theta} \tan \phi-\sqrt{\operatorname{det}(\mathbb{G})}\left(\frac{d g_{\theta \theta}}{d \phi} \tan \phi+g_{\theta \theta}\left(1+\tan ^{2} \phi\right)\right)}{g_{\theta \theta}^{2} \tan ^{2} \phi} \\
& =\frac{g_{\theta \theta} \frac{d g_{\phi \phi}}{d \phi}+g_{\phi \phi} \frac{d g_{\theta \theta}}{d \phi}-2 g_{\theta \phi} \frac{d g_{\theta \phi}}{d \phi}}{2 \operatorname{det}(\mathbb{G}) g_{\theta \theta} \tan \phi}-\frac{\frac{d g_{\theta \theta}}{d \phi} \tan \phi+g_{\theta \theta}\left(1+\tan ^{2} \phi\right)}{g_{\theta \theta}^{2} \tan ^{2} \phi}
\end{aligned}
$$


Using (36), the determinant of the manifold metric $\mathbb{G}$ is found to be

$$
\begin{aligned}
& \operatorname{det}(\mathbb{G})=\pi^{4} \cos ^{2} \phi \sin ^{2} \phi \\
& \cdot\left\{\left(c_{3} \cos ^{2} \theta-2 c_{2} \cos \theta \sin \theta+c_{1} \sin ^{2} \theta\right)\right. \\
& \cdot\left(c_{1} \cos ^{2} \theta+2 c_{2} \cos \theta \sin \theta+c_{3} \sin ^{2} \theta\right) \\
&\left.-\left(c_{2}\left(\sin ^{2} \theta-\cos ^{2} \theta\right)+\left(c_{1}-c_{3}\right) \cos \theta \sin \theta\right)^{2}\right\} .
\end{aligned}
$$

Let us denote the first term inside the braces as $X$ and the second as $Y$ i.e.,

$$
\left\{\begin{aligned}
X= & \left(c_{3} \cos ^{2} \theta-2 c_{2} \cos \theta \sin \theta+c_{1} \sin ^{2} \theta\right) \\
& \left(c_{1} \cos ^{2} \theta+2 c_{2} \cos \theta \sin \theta+c_{3} \sin ^{2} \theta\right) \\
Y= & \left(c_{2}\left(\sin ^{2} \theta-\cos ^{2} \theta\right)+\left(c_{1}-c_{3}\right) \cos \theta \sin \theta\right)^{2} .
\end{aligned}\right.
$$

The above expressions can be simplified to

$$
\left\{\begin{aligned}
X= & c_{1} c_{3} \chi^{4}-2 c_{1} c_{2} \chi^{3} \sigma+c_{1}^{2} \chi^{2} \sigma^{2}+2 c_{2} c_{3} \chi^{3} \sigma-4 c_{2}^{2} \chi^{2} \sigma^{2} \\
& +2 c_{1} c_{2} \chi \sigma^{3}+c_{3}^{2} \chi^{2} \sigma^{2}-2 c_{2} c_{3} \chi \sigma^{3}+c_{1} c_{3} \sigma^{4} \\
Y= & c_{2}^{2} \chi^{4}-c_{1} c_{2} \chi^{3} \sigma+c_{2} c_{3} \chi^{3} \sigma-c_{2}^{2} \chi^{2} \sigma^{2}-c_{1} c_{2} \chi^{3} \sigma+c_{1}^{2} \chi^{2} \sigma^{2} \\
& -c_{1} c_{3} \chi^{2} \sigma^{2}+c_{1} c_{2} \chi \sigma^{3}+c_{2} c_{3} \chi^{3} \sigma-c_{1} c_{3} \chi^{2} \sigma^{2}+c_{3}^{2} \chi^{2} \sigma^{2} \\
& -c_{2} c_{3} \chi \sigma^{3}-c_{2}^{2} \chi^{2} \sigma^{2}+c_{1} c_{2} \chi \sigma^{3}-c_{2} c_{3} \chi \sigma^{3}+c_{2}^{2} \sigma^{4}
\end{aligned}\right.
$$

where $\chi=\cos \theta$ and $\sigma=\sin \theta$, and hence, $\chi^{2}+\sigma^{2}=1$.

Hence

$$
\begin{aligned}
\operatorname{det}(\mathbb{G})= & \pi^{4} \cos ^{2} \phi \sin ^{2} \phi(X-Y) \\
= & \pi^{4} \cos ^{2} \phi \sin ^{2} \phi \\
& \cdot\left(\left(c_{1} c_{3}-c_{2}^{2}\right) \chi^{4}+2\left(c_{1} c_{3}-c_{2}^{2}\right) \chi^{2} \sigma^{2}\right. \\
& \left.\quad+\left(c_{1} c_{3}-c_{2}^{2}\right) \sigma^{4}\right) \\
= & \pi^{4} \cos ^{2} \phi \sin ^{2} \phi\left(c_{1} c_{3}-c_{2}^{2}\right)\left(\chi^{2}+\sigma^{2}\right)^{2} \\
= & \pi^{4} \cos ^{2} \phi \sin ^{2} \phi\left(c_{1} c_{3}-c_{2}^{2}\right) \\
= & \frac{\pi^{4}}{4} \operatorname{det}(\mathbb{L}) \sin ^{2}(2 \phi) .
\end{aligned}
$$

\section{Proof of (22)}

The angle $\psi_{0}$ can be found through points of extremum curvatures; hence, since the curvature of the development is equal to $\kappa_{g \theta}$, the geodesic curvature of the $\theta$ curves of the planar array manifold is differentiated with respect to $\theta$ and equated to zero

$$
\begin{aligned}
\frac{d \kappa_{g \theta}}{d \theta}=0 & \\
\Leftrightarrow & \frac{d\left(c_{3} \cos ^{2} \psi_{0}-2 c_{2} \cos \psi_{0} \sin \psi_{0}+c_{1} \sin ^{2} \psi_{0}\right)^{-(3 / 2)}}{d \psi_{0}}=0 \\
\Leftrightarrow & \left(c_{3} \cos ^{2} \psi_{0}-2 c_{2} \cos \psi_{0} \sin \psi_{0}+c_{1} \sin ^{2} \psi_{0}\right)^{-(5 / 2)} \\
& \cdot\left(\left(c_{1}-c_{3}\right) \sin \left(2 \psi_{0}\right)-2 c_{2} \cos \left(2 \psi_{0}\right)\right)=0 \\
& \Leftrightarrow\left\{\begin{array}{l}
c_{3} \cos ^{2} \psi_{0}-2 c_{2} \cos \psi_{0} \sin \psi_{0}+c_{1} \sin ^{2} \psi_{0}=0 \\
\text { or } \\
\left(c_{1}-c_{3}\right) \sin \left(2 \psi_{0}\right)-2 c_{2} \cos \left(2 \psi_{0}\right)=0 .
\end{array}\right.
\end{aligned}
$$

Considering the first equation in the list

$$
c_{3} \cos ^{2} \psi_{0}-2 c_{2} \cos \psi_{0} \sin \psi_{0}+c_{1} \sin ^{2} \psi_{0}=0
$$

the discriminant gives $\Delta^{2}=-4 \operatorname{det}(\mathbb{L})<0$, and hence, the first equation has no solution.

The second equation gives $\tan \left(2 \psi_{0}\right)=2 c_{2} /\left(c_{1}-c_{3}\right)$, and hence, $\psi_{0}=(1 / 2) \tan ^{-1}\left(2 c_{2} /\left(c_{1}-c_{3}\right)\right)$.

\section{REFERENCES}

[1] A. Manikas, R. Karimi, and I. Dacos, "Study of the detection and resolution capabilities of one-dimensional array of sensors by using differential geometry," Proc. Inst. Elect. Eng., Radar, Sonar, Navig., vol. 141, no. 2, pp. 83-92, Apr. 1994.

[2] H. R. Karimi and A. Manikas, "Manifold of a planar array and its effects on the accuracy of direction-finding systems," Proc. Inst. Elect. Eng., Radar, Sonar, Navig., vol. 143, no. 6, pp. 349-357, Dec. 1996.

[3] A. Manikas, A. Alexiou, and H. R. Karimi, "Comparison of the ultimate direction-finding capabilities of a number of planar array geometries," Proc. Inst. Elect. Eng., Radar, Sonar, Navig., vol. 144, no. 6, pp. 321-329, Dec. 1997.

[4] A. Manikas and C. Proukakis, "Modeling and estimation of ambiguities in linear arrays," IEEE Trans. Signal Processing, vol. 46, pp. 2166-2179, Aug. 1998

[5] A. Manikas, C. Proukakis, and V. Lefkaditis, "Investigative study of planar array ambiguities based on "hyperhelical' parameterization," IEEE Trans. Signal Processing, vol. 47, pp. 1532-1541, June 1999.

[6] R. O. Schmidt, "Multiple emitter location and signal parameter estimation," IEEE Trans. Antennas Propagat., vol. AP-34, pp. 276-280, Mar 1986.

[7] R. O. Schmidt, "Multilinear array manifold interpolation," IEEE Trans. Signal Processing, vol. 40, pp. 857-866, Apr. 1992.

[8] I. Dacos and A. Manikas, "Estimating the manifold parameters of onedimensional arrays of sensors," J. Franklin Inst. (Eng. Appl. Math.), vol 332B, no. 3, pp. 307-322, May 1995

[9] T. J. Willmore, An Introduction to Differential Geometry. Oxford, U.K.: Oxford Univ. Press, 1959.

[10] A. Gray, Modern Differential Geometry of Curves and Surfaces. College Park, MD: Univ. of Maryland Press, 1993.

[11] D. Martin, Manifold Theory: An Introduction for Mathematical Physicists. New York: Ellis Horwood, 1991.

[12] H. Guggenheimer, Differential Geometry. New York: McGraw-Hill, 1963.

[13] M. Spivak, A Comprehensive Introduction to Differential Geometry. Berkeley, CA: Publish or Perish, 1979.

[14] L. P. Eisenhart, Riemannian Geometry. Princeton, NJ: Princeton Univ. Press, 1949

[15] H. R. Karimi and A. Manikas, "Cone-angle parametrization of the array manifold in df systems," J. Franklin Inst. (Eng. Appl. Math.), vol. 335B, no. 2, pp. 375-394, 1998 .

[16] I. Dacos and A. Manikas, "Eliminating the quantization problem in signal subspace techniques," IEICE Trans. Commun., vol. E78-B, no 11, pp. 1458-1467, Nov. 1995.

[17] _ , "Investigating the manifold parameters of a non linear array of omnidirectional sensors-Part ii: Specialization to planar arrays of arbitrary geometry," Imperial Coll. Sci. Technol. Medicine, London, U.K., Unclassified-Research Rep. AM-91-5, Sept. 1991.

[18] N. H. Dowlut and A. Manikas, "A polynomial rooting approach to superresolution array design," IEEE Trans. Signal Processing, vol. 48, pp. $1559-1569$, June 2000

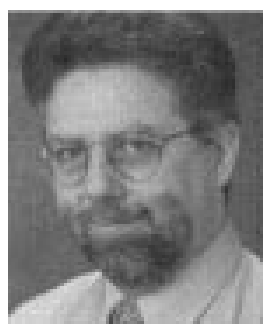

Athanassios Manikas (M'88) received the Ph.D. and D.I.C. degrees from Imperial College, University of London, London, U.K., in 1988 and was appointed Lecturer at Imperial College the same year.

$\mathrm{He}$ is now a Reader in digital communications with the Department of Electrical and Electronic Engineering, Imperial College of Science, Technology, and Medicine, London. He has published an extensive set of journal and conference papers relating to his research interests, which are in the general area of digital communication and signal processing, where he has developed a wide and deep interest in the topic of super-resolution array processing and the application of array theory to communication systems and problems. His major concern is with the fundamental problems of arrays and the role they play in the development of future digital communication systems aiming at exploring and exploiting new spectrum and space efficiency frontiers by integrating arrays with diverse broadband wireless communication systems (current and future) with a view to increasing the system capacity without compromising quality of service. He is currently the Deputy Head of the Communications and Signal Processing Research Group at Imperial College, and over the last nine years, he has held a number of important research consultancies for the EU, industry, and government organizations.

Dr. Manikas is a corporate member of IEE and a Chartered Electrical Engineer. 


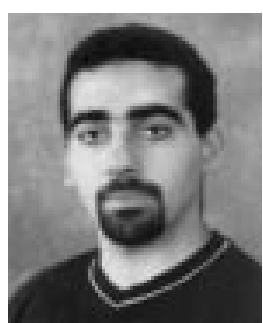

Adham Sleiman (S'99) received the M.E. degree (with honors) in electrical and electronic engineering in 1998 from Imperial College, University of London, London, U.K. In 1998, he was awarded a research scholarship from the Department of Electrical and Electronic Engineering at Imperial College and has since that date been pursuing the Ph.D. degree with the Communications and Signal Processing Research Group.

He studied telecommunications for one year at the Ecole Nationale Supérieure des Télécommunications (E.N.S.T.), Paris, France, in 1997. His main research interests are in the area of array design through the use of mathematical tools such as differential geometry, array processing techniques, ambiguity issues in direction finding, and the use of antenna arrays in mobile communication systems of the third generation and beyond.

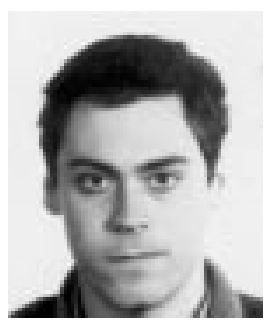

Ioannis Dacos received M.Sc. degree (with distinction) in communications engineering in 1988 and the Ph.D. and D.I.C. degrees in 1992, all from the Department of Electrical and Electronic Engineering, Imperial College of Science Technology and Medicine, London, U.K.

$\mathrm{He}$ is with Greek Telecom (OTE) and is a Lecturer with the Communication Engineering Department, Hellenic Air Force Academy, Athens, Greece. His research interests are in the area of array processing and superresolution spatio-temporal spectral estimation as well as in the general area of digital communications.

Dr. Dacos is a member of the Technical Chamber of Greece. 